\title{
118. Sur le Théorème de Plancherel
}

Par Shin-ichi MATSUSHITA

(Comm. by K. KunUGI, M.J.A., July 12, 1954)

1. Le but du présent article est d'exposer une formule généralisée de Plancherel dans un groupe localement compact, qui n'est nécessairement ni unimodulaire ni abélien; le même subjet fut déjà étudié par l'auteur, toutefois, un lapsus s'est glissé dans l'énoncé du théorème central, qu'il faut remplacer par Théorème $C$ dans ce travail. ${ }^{1)}$ Une démonstration très simple du théorème de Plancherel pour un groupe abélien sera donnée dans le paragraphe 5.

2. Soit $G$ un groupe localement compact muni de la mesure de Haar à gauche $d x$ et de la fonction mondulaire $\rho(x)$ telle que $d x^{-1}=\rho(x) d x:^{2)}$ l'élément neutre sera noté $e$. On notera $L^{0}(\cdot)$ l'espace vectoriel normé des fonctions continues à support compact définies dans un espace localement compact $(\cdot)$, par $L^{+}(\cdot)$ le cône positif de $L^{0}(\cdot)$, et par $L_{\infty}(\cdot)$ l'adhérence uniforme de $L^{0}(\cdot)$, qui se compose des $f$ telles que $f(x)$ tende vers zéro lorsque $x$ tend vers l'infini. On désignera encore par $L(G)$ (resp. $L^{2}(G)$ ) l'ensemble des fonctions sommables (resp. de carré sommable) sur $G$ pour $d x$; $L(G)$ constitue alors une algèbre de Banach par rapport au produit de composition, $f g(x)=\int_{G} f(y) g\left(y^{-1} x\right) d y$.

En désignant par $\stackrel{G}{P}(G)$ (ou $P_{0}(G)$ ) l'ensemble de toutes les fonctions continues (resp., de plus, des normes $\leqq 1$ ) de type positif, on voit facilement que $P_{0}(G)$ forme un sous-ensemble convexe, faiblement compact de $P(G)$ et donc possède l'ensemble des points extrémaux, noté $V$, qui engendrent linéairement $P_{0}(G)$ tout entier: on appelle espace de caractères de $G$, noté $V_{0}$, l'ensemble défini par $V^{a}$ $-\theta$, ou $V^{a}$ est l'adhérence faible de $V$ et $\theta$ fonction zéro. Autrement dit, $V_{0}$ est l'ensemble des fonctions élémentaires (c'est-à-dire, fonctions de $V-\theta$ ) et leurs limites faibles distinctes de $\theta$. Notons alors le fait important;

Théorème A (M. Godement). ${ }^{3)}$ A toute fonction $g(x)$ de $P(G)$ est associée au moins une (et une seule, si $G$ est abélien) mesure $d \mu_{g}$ définie sur $V_{0}$, bornée positive et de la norme $\left\|\mu_{g}\right\|=\int_{V_{0}} d \mu_{g}=g(e)$, telle qu'on ait pour toute $f$ de $L^{0}(G)$,

1) S. Matsushita: C. R. Acad. Sci., Paris. 955-957, 1056-1057 (1953). Dans ces Notes, on doit employer $\lim _{\lambda} \int d_{\mu_{f_{\lambda}}}$ au lieu de $\int d \varphi$ (voir le paragraphe 4).

2) On aura soin de ne pas confondre notre $\rho(x)$ avec celle $\Delta(x)$ au sens de M. Weil; on a $\rho(x)=\Delta(x)^{-1}$

3) R. Godement: Trans. Amer. Math. Soc., 6, 3 (1948). 


$$
\int_{G} \overline{g(x)} f(x) \sqrt{\rho(x)} d x=\int_{V_{0}} \hat{f}(\varphi) d \mu_{g}(\varphi),
$$

$$
\hat{f}(\varphi)=\int_{V_{0}} \overline{\varphi(x)} f(x) \sqrt{\rho(x) d x} \quad \text { pour } \varphi \in V_{0} .
$$

Si, pour chaque $x \in G$, la fonction $\varphi(x)$ est continue sur $V_{0}$, on a

$$
g(x)=\int_{V_{0}} \varphi(x) d \mu_{g}(\varphi)
$$

Ce théorème (théorème généralisé de Bochner) peut être démontré sans peine à l'aide du théorème de Riesz-Markoff; en appliquant la formule de Fubini à (2. 1), on obtiendra aisément (2. 3).

La fonction $\hat{f}(\varphi)$ définie par (2.2) ci-dessus, qui est évidemment continue sur $V_{0}$, s'appelle la transformée de Fourier de $f$.

Corollaire. Si $f$ et $g$ appartiennent ̀̀ $P(G) \cap L^{0}(G)$, il en vient comme une conséquence immédiate de (2. 1) que

$$
\int_{V_{0}} \hat{f}(\varphi) d \mu_{g}(\varphi)=\int_{V_{0}} \hat{g}(\varphi) d \mu_{f}(\varphi) .
$$

Désignant par $\Pi(G)$ l'enveloppe linéaire complexe de $P(G)$, et posant $\mu_{g}=\mu_{g_{1}}-\mu_{g_{2}}+i\left(\mu_{g_{3}}-\mu_{g_{4}}\right)$ pour $g \in \Pi(G)$ avec $g=g_{1}-g_{2}+i\left(g_{3}-g_{4}\right)$, $g_{k} \in P(G), \quad k=1,2,3,4$, on déduit du théorème $\mathrm{A}$ une formule étendue du théorème classique de Parseval;

Théorème B. A toute fonction $g(x)$ de $\Pi(G)$ est associée au moins une mesure complexe $\mu_{g}$ définie sur $V_{0}$, telle qu'on ait pour toute $f$ de $L^{0}(G)$

$$
\int_{G} \overline{g(x)} f(x) V \rho(x) d x=\int_{V_{0}} \hat{f}(\varphi) \overline{d \mu_{g}}(\varphi),{ }^{4)}
$$

et $\int_{V_{0}} d \mu_{g}=\left\|g_{1}\right\|-\left\|g_{2}\right\|+i\left(\left\|g_{3}\right\|-\left\|g_{4}\right\|\right)=g(e)$. De plus, sous la même condition que dans (1.3), on a

$$
g(x)=\int_{V_{0}} \varphi(x) d \mu_{g}(\varphi)
$$

3. Pour chaque voisinage compact $U$ de $e$, il y a une fonction $g_{U}$ de $L^{+}(G)$ qui a son support dans $U$ et puis est symétrique, c'està-dire $g_{U}\left(x^{-1}\right)=g_{U}(x)$; en effet, soit $h \in L^{+}(G)$ de la forme $h=h^{*}$ (où $\left.h^{*}(x)=h\left(x^{-1}\right) \rho(x)\right)$ et avec son support dans $U_{0}$ tel que $U_{0} U_{0}^{-1} \subset U$ (l'existence exacte de telle $h$ est évidente), alors $g_{U}(x)=h(x) / V \rho(x)$ satisfait aux conditions ci-dessus. En outre, supposons toujours que $\int_{G} g_{U}(x) d x=1$.

Ceci étant, posant $f_{U}(x)=\int_{G} g_{U}(y) g_{U}(x y) d y, f_{U}$ est alors à la fois i) positive ii) de type positif et iii) $\int_{G} f_{U}(x) d x=1$. Le support de $f_{U}$

4) $d \mu$ désigne la mesure conjuguée de $d \mu$. 
est contenu dans $U$.

$\boldsymbol{A}_{U}$ désignant l'ensemble de ces $f_{U}$ pour chaque $U$, on voit facilement que les $\boldsymbol{A}_{U}$ costituent une base du filtre $\boldsymbol{\Lambda}$ sur $P(G) \cap L^{0}(G)$, suivant lequel la mesure $f_{U} d x$ converge étroitement vers la masse +1 placée en $e$; alors les transformées de Fourier des $f_{U}$ convergent vers la constante un sur $V_{0}$ pour la topologie de la convergence compacte.

4. Pour $f, g \in L^{0}(G)$, nous posons

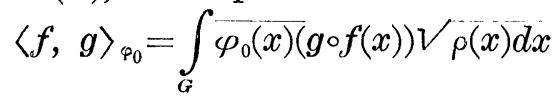

pour $\varphi_{0} \in V_{0}$, où

$$
g \circ f(x)=\int_{G} \overline{g(u)} f(x u) d u \text {. }
$$

Il est clair que $f \circ f \in P(G)$ et donc $g \circ f \in \Pi(G)$ : nous avons alors $\left\langle f, g_{\rho_{0}}\right\rangle=\left\langle g, f_{\varphi_{0}}\right\rangle$ et $\langle f, f\rangle_{\varphi_{0}} \geqq 0$.

Les $g \in L^{0}(G)$ telles que $\langle g, g\rangle_{\varphi_{0}}=0$ constituent un sous-espace $N_{\varphi_{0}}$ de $L^{0}(G)$; alors, $H_{\varphi_{0}}^{0} \equiv L^{0}(G) / N_{\varphi_{0}}$ forme un espace pré-hilbertien par rapport au produit scalaire

$$
\left(X_{f}^{\varphi}, X_{g}^{\varphi}\right)_{\varphi}=\langle f, g\rangle_{\varphi},
$$

où $X_{f}^{\varphi}, X_{g}^{\varphi} \in H_{\varphi}{ }^{0}$ et $f \in X_{f}^{0}, g \in X_{g}^{0}$ : Si $G$ est abélien, $H_{\varphi}{ }^{0}$ est à une dimension, et alors $X_{f}^{\text {s }}$ peut être confondant avec la valeur $\hat{f}(\varphi){ }^{5)}$

Considérons maintenant la somme directe $H_{V_{0}}{ }^{0} \equiv \sum \oplus H_{\varphi^{0}}{ }^{0}$, dont chaque élément $\tilde{f}$ est de la forme telle que $\tilde{f} \equiv \sum_{\varphi \in V_{0}} \underset{X_{f}^{\varphi}}{\mathcal{\varphi}_{0}}$ pour $f \in$ $L^{0}(G)$; en posant

$$
(\tilde{f}, \tilde{g})_{V_{0}}=\lim _{\lambda} \int_{V_{0}}\left(X_{f}^{\varphi}, X_{g}^{\varphi}\right)_{p} d \mu_{f_{\lambda}}(\varphi)
$$

pour $f_{\lambda} \in \Lambda$ (voir le paragraph précédent), on voit facilement que ce produit scalaire est bien défini; en effet, parce que $g \circ f \in \Pi(G)$ $\bigcap L^{0}(G)$, on voit d'apèrs Théorème $\mathrm{B}$ que

$$
\begin{aligned}
\int_{V_{0}}\left(X_{f}^{\varphi}, X_{g}^{\varphi}\right)_{\varphi} d \mu_{f_{\lambda}}(\varphi) & =\int_{V_{0}} \widehat{g \circ} f(\varphi) d \mu_{f_{\lambda}}(\varphi) \\
& =\int_{G} g \circ f(x) f_{\lambda}(x) \sqrt{\rho(x)} d x \rightarrow g \circ f(e)=\int_{V_{0}} d \mu_{g} \circ f(\varphi),
\end{aligned}
$$

malgré que $\mathrm{d} \mu_{f_{\lambda}}$ ne soient pas uniquement déterminées.

De plus, on a $\|\tilde{f}\|_{V_{0}}^{2}=(\tilde{f}, \tilde{f})_{V_{0}}=f \circ f(e)=\int_{G}|f|^{2} d x$; ainsi, $H_{V_{0}}^{0}$ normé par cette $\|\tilde{f}\|_{V_{0}}$ est isométriquement isomorphe à $L^{0}(G)$ considéré comme un sous-espace de $L^{2}(G)$; en complétant $H_{V_{0}}^{0}$ pour cette norme, on obtient un espace hilbertien $H_{V_{0}}$ qui est isomorphe à $L^{2}(G)$ tout entier, car $L^{0}(G)$ est partout dense dans $L^{2}(G)$.

Théorème C. Les deux espaces hilbertiens $H_{V_{0}}$ et $L^{2}(G)$ sont iso-

5) Si $G$ est abélien, $\left(X_{f}^{\varphi}, X_{g}^{\varphi}\right)_{p}=\hat{f}(\varphi) \overline{\hat{g}(\varphi)}$. 
métriquement isomorphes l'un à l'autre. Si $G$ est abélien, on a $H_{V_{0}}$ $=L^{2}(\hat{G})$ et

$$
(\tilde{f}, \tilde{g})_{V_{0}}=\int_{\hat{G}} \hat{f}(\varphi) \ddot{\hat{g}}(\varphi) d \varphi,
$$

où $\hat{G}\left(=V_{0}\right)$ est le groupe dual de $G$ et do désigne une mesure de Haar sur $\hat{G}$.

5. En effet, si $G$ est abélien, on a

$$
\hat{f_{1}}(\varphi) d \mu_{f_{2}}(\varphi)=\hat{f}_{2}(\varphi) d \mu_{f_{1}}(\varphi)
$$

pour deux fonctions quelconques $f_{1}, f_{2}$ de $P(G) \cap L^{0}(G)$, car on a

$$
\begin{aligned}
\int_{V_{0}} \hat{g}(\varphi) \hat{f}_{i}(\varphi) d \mu_{f_{j}}(\varphi) & =\int_{V_{0}} \hat{g f_{i}}(\varphi) d \mu_{f_{j}}(\varphi) \\
=\int_{G} g f_{i}(x) f_{j}(x) d x & =\int_{G} g f_{j}(x) f_{i}(x) d x
\end{aligned}
$$

pour toute $g \in L(G) ; i, j=1$, 2, et l'ensemble de toutes les $\hat{g}, g \in L(G)$, est partout dense dans $L_{\infty}(\hat{G})$ en vertu du théorème connu de M. Stone.

Or, l'ensemble des mesures $d \mu_{f_{\lambda}}, \lambda \in \Lambda$, est borné au sens de M. Bourbaki, ${ }^{6)}$ qui est alors relativement compact pour la topologie vague; par suite, il existe une sous-famille des $f_{\lambda^{\prime}}$, qui converge vaguement vers une mesure $d \varphi$ sur $G$, c'est-à-dire $\hat{f}(\varphi) d \varphi=\lim _{\lambda^{\prime}}$ vag. $\hat{f}(\varphi) d \mu_{f_{\lambda^{\prime}}}$ pour toute $f \in L(G)$. vient

D'autre part, on a $\lim _{\lambda}$ vag. $\hat{f} d \mu_{f_{\lambda}}=\lim _{\lambda}$ vag. $\hat{f}_{\lambda} d \mu_{f}=d \mu_{f}$, d'où il

$$
d \mu_{f}=\hat{f} d \varphi \quad \text { pour toute } f \in L(G) \cap \Pi(G) .
$$

Clairement, $d \varphi$ est positive et n'est pas identiquement zéro, de plus, est invariante par translation, car $\int_{\hat{G}} \hat{g}(\varphi) \hat{f}(\varphi) d\left(\varphi_{0}^{-1} \varphi\right)$ $=\int_{\hat{G}} \hat{g}\left(\varphi_{0} \varphi\right) \overline{\hat{f}}\left(\varphi_{0} \rho\right) d \varphi=\int_{G}\left(g^{\prime}(x) \varphi_{0}(x)\right) \overline{\left(f(x) \varphi_{0}(x)\right)} d x=\int_{G} g^{\prime}(x) \overline{f(x)} d x$ $=\int_{\hat{\hat{G}}} \hat{g}(\varphi) \hat{f}(\varphi) d(\varphi)$ pour toutes $f \in \Pi(G) \cap L(G), g \in L(G)$; d'où $f_{\lambda}(\varphi) d\left(\varphi_{0}^{-1} \varphi\right)=f_{\lambda}(\varphi) d \varphi$ pour tout $\lambda \in \Lambda$, et donc $d \varphi_{0}^{-1} \varphi=d \varphi$.

Finalement, $H_{\hat{G}}^{0}$ est dense dans $L^{2}(\hat{G})$; en effet, soit $\tilde{f}_{0} \in L^{+}(\hat{G})$, la mesure bornée $\tilde{f}_{0} d \varphi$ définit une fonctionnelle linéaire $\tau \tilde{f}_{0}$ sur $L^{2}(G)$ de la manière suivante: pour toute $g \in \Pi(G) \cap L(G)$, on a

$$
\tau \tilde{f}_{0}(g)=\int_{\hat{G}} \hat{g}(\varphi) \tilde{f}_{0}(\varphi) d(\varphi),
$$

qui est en module $\leqq\|\hat{g}\|_{2} \cdot\left\|\tilde{f}_{0}\right\|_{2}=\|g\|_{2} \cdot\left\|\tilde{f}_{0}\right\|_{2}$, d'où $\tau_{f_{0}}$ définit un élé-

6) N. Bourbaki: Intégration, 62-63 (1952). 
ment $f_{0}$ de $L^{2}(G)$ qui correspond à $\tilde{f_{0}}$, car $\Pi(G) \cap L(G)$ est dense dans $L^{2}(G)$. Enfin, $L^{0}(G) \subset H_{\hat{G}}$, d'où résulte que $H_{\hat{G}}=L^{2}(\hat{G})$.

6. Voici en passant une application de l'étude ci-devant: soit $\breve{L}(G)$ l'ensemble des fonctions continues et sommables pour la mesure $\sqrt{\rho(x)} d x$, alors pour chaque $\varphi \in V_{0}$ le produit scalaire

$$
\begin{aligned}
& \langle f, g\rangle_{\text {P }} \\
& =\int_{G} \int_{G} \varphi\left(x y^{-1}\right) f(x) \overline{g(y)} \sqrt{\rho(x)} d x \sqrt{\rho(y) d y}
\end{aligned}
$$

est bien défini pour $f, g \in \breve{L}(G)$. Lorsqu'on considère $f, g$ de $L^{0}(G)$ qui est évidemment contenu dans $\breve{L}(G)$, ce produit-ci s'identifie à celui défini dans (4. 1), comme on le vérifie directement.

Or, mettant en parallèle au paragraphe 4 , ce produit permet de définir un espace pré-hilbertien $\breve{H}_{\varphi}^{0} \equiv \breve{L}(G) / \breve{N}_{\varphi}$, où $\breve{N}_{\varphi}$ désigne un sous-espace de $L(G) \operatorname{des} f$ telles qu'on ait $\langle f, f\rangle_{\varphi}=0$, et l'on obtient alors un espace hilbertien $\breve{H}_{\varphi}$ en complétant $\breve{H}_{\varphi}{ }^{0}$.

Dans $\breve{H}_{\varphi}$, la famille filtrante des $X_{f_{\lambda}^{\varphi}}$ pour $\lambda \in \Lambda$ converge faiblement suivant $\Lambda$ vers un élément $X_{0}^{\varphi}$ tel que $\left(X_{g}^{\varphi}, X_{0}^{\varphi}\right)_{\varphi}=\hat{g}(\varphi)$ pour toute $g \in \breve{L}(G)$; en effet, on voit que

$$
\begin{aligned}
& \left(X_{f_{\lambda}^{\varphi}}^{\varphi} \quad X_{g}^{\varphi}\right)_{\varphi}=\int_{G} f_{\lambda}(x) \int_{G} \varphi(y) \overline{g(y x)} \sqrt{\rho(y)} d y d x \\
& \left.\rightarrow \quad \int_{G} \varphi(y) \overline{g(y)} \sqrt{\rho(y)} d y=\overline{g(\varphi)} \quad \text { (avec } \lambda\right),
\end{aligned}
$$

d'où l'égalité ci-dessus. Alors, l'intégral dans le second membre de (4.4) est aussi bien définie pour $\left(X_{g}^{\varphi}, X_{0}^{\varphi}\right)_{\varphi}$, ce qui s'écrit

$$
\lim _{\lambda} \int_{V_{0}}\left(X_{g}^{\varphi}, X_{0}^{\varphi}\right)_{\varphi} d \mu_{f_{\lambda}}(\varphi)=\lim _{\lambda} \int_{V_{0}} \hat{g}(\varphi) d \mu_{f_{\lambda}}(\varphi)=g(e) .
$$

Soit $g_{s}(\cdot)=g\left(\cdot s^{-1}\right) \sqrt{\rho(s)}$; pour toute $g \in \breve{L}(G)$, l'opération linéaire $g \rightarrow g_{s}$ définit un opérateur unitaire $U_{s}$ dans chaque $\breve{H}_{\varphi}$, qui vérifie $\left(X_{g s}^{\varphi}, X_{0}^{\varphi}\right)_{\varphi}=\left(U_{s} X_{g}^{\varphi}, X^{\circ \varphi}\right)_{\varphi}=\left(X_{g}^{\varphi}, U_{s^{-1}} X_{0}^{\varphi}\right)_{\uparrow}$. D'où on déduit aussitôt que

$$
g(x)=\lim _{\lambda} \int_{V_{0}}\left(X_{g}^{\varphi}, U_{x} X_{0}^{\varphi}\right)_{\varphi} d \mu_{f_{\lambda}}(\varphi),
$$

ce qui n'est autre que la formule étendue d'inversion de Fourier, vu qu'on a $\hat{g}(\varphi)=\left(X_{g}^{\varphi}, X_{0}^{\varphi}\right)_{\varphi}$ et $\varphi(x)=\left(X_{0}^{\varphi}, U_{x} X_{0}^{\varphi}\right)_{\varphi}$. Si $G$ est abélien, ${ }^{7)}$ on a $\left(X_{g}^{\varphi}, U_{x} X_{0}^{\varphi}\right)_{\varphi}=\varphi(x) \hat{g}(\varphi)$; cela signifie que (6. 2) entraîne

$$
g(x)=\int_{\hat{\theta}} \varphi(x) \hat{g}(\varphi) d \varphi, \quad \text { pour } \quad g \in L(G) \cap \Pi(G),
$$

en tenant compte du paragraphe précédent.

7) Dans le cas où $G$ est abélien ou plus généralement unimodulaire, on a $\breve{L}(G)$ $=L(G) \cap C(G)$. 